\title{
Pre-exposure enhances recovery of conditioned responding after extinction
}

\author{
Hannah Rosenberg • Nathan M. Holmes • \\ Justin A. Harris $\cdot$ R. Frederick Westbrook
}

Published online: 15 February 2011

(C) Psychonomic Society, Inc. 2011

\begin{abstract}
Four experiments used a within-subjects design with rats to study the effects of preexposure on the restoration of fear responses (freezing) to an extinguished conditioned stimulus (CS). In each experiment, rats were preexposed to one CS (A), but not to another (B), and then were exposed to pairings of each of these CSs with an aversive unconditioned stimulus (US). In each experiment, there was less freezing to A than to B across extinction, showing a latent inhibitory effect of preexposure. There was no differential recovery to A and $\mathrm{B}$ following either a US reexposure (Experiment 1) or a delay interval (Experiment 2). However, when a delay interval included US reexposure, there was greater recovery to the preexposed CS, A, than to the nonpreexposed CS, B (Experiments 1, 3, and 4). These results suggest that the effects of US reexposure and delay combine to affect recovery from the depressive effects of CS-alone exposure. The results are consistent with the view that US reexposure produces better mediated conditioning of CSs that are strongly associated with the context. The results may additionally reflect an effect of preexposure on the learning produced by extinction.
\end{abstract}

Keywords Pavlovian conditioning $\cdot$ Pre-exposure $\cdot$ Latent inhibition $\cdot$ Extinction $\cdot$ Rats

Latent inhibition of Pavlovian conditioned responding is a well-documented phenomenon (Lubow \& Moore, 1959).

H. Rosenberg $\cdot$ N. M. Holmes $\cdot$ R. F. Westbrook $(\bowtie)$

School of Psychology The University of New South Wales,

Sydney 2033, Australia

e-mail: f.westbrook@unsw.edu.au

J. A. Harris

School of Psychology, The University of Sydney,

Sydney 2006, Australia
Subjects in one group, but not in another, are repeatedly exposed to a stimulus in the absence of any other scheduled event. Then subjects in both groups are exposed to a signaling relation between that stimulus and an unconditioned stimulus (US). The responding (aversive or appetitive) elicited by the now-conditioned stimulus (CS) in the preexposed subjects is depressed, relative to that shown by the nonpreexposed subjects. The preexposure is said to have latently inhibited subsequent conditioned responding. Latent inhibition has also been observed in within-subjects designs where subjects are preexposed to one, but not to another, CS and then to a signaling relation between each of the CSs and a US. The responding elicited by the preexposed CS is again depressed, relative to that elicited by the nonpreexposed CS (Holmes \& Harris, 2010; Killcross \& Robbins, 1993; Rescorla, 2002). Thus, latent inhibition is an inability of a preexposed stimulus to elicit conditioned responding, rather than an inability of preexposed subjects to exhibit such responding.

The conditions required to produce latent inhibition are relatively simple: nonreinforced exposures to the CS-to-be. In contrast, there has been much debate concerning what is learned during the phase of stimulus-alone exposures that produces the subsequent retardation in conditioned performance (for a review, see Holmes \& Harris, 2010). One proposal is that during preexposure, subjects learn a CS-nothing or CS-no-event association (Bouton, 1991, 1993; Hall \& Honey, 1989; Hall \& Rodriguez, 2010; Lubow et al., 1981; Westbrook \& Bouton, 2010). For example, Hall and Rodriguez (2010) argued that salient events rarely occur in isolation and that subjects thus enter preexposure expecting that the events presented will be followed by some outcome (e.g., loud sounds are accompanied by the appearance of their causes). Hence, the discrepancy between what actually occurs (no event) and that expected on the basis of prior experience 
(salient events rarely occur in isolation) results in subjects' forming either a general CS-no event association or one that is specific to their current motivational state (Killcross \& Balleine, 1996). Moreover, since salient stimuli (such as a noise) are followed by outcomes elsewhere (in the home cage), the CS-no-event association formed across preexposure is encoded by the situational cues present, leading to latent inhibition when the $\mathrm{CS}$ is subsequently encountered in their presence and to conditioned responding in their absence. Therefore, according to this view, the generalized expectancy of some event elicited by the preexposed CS is canceled by the formation of a CS-no-event association. This almost exactly describes the conditions that produce extinction of CS-US learning. Extinction refers to the decrement in conditioned performance that results from postconditioning CS-alone exposures. This decrement is not due to erasure of the original CS-US association but, instead, appears to be due to acquisition of a CS-no-US association that counters expression of the original CS-US association. Thus, latent inhibition and extinction are both consequences of CS-alone exposures, and both appear to involve some form of inhibitory learning: a CS-no-event association in the case of preexposure versus a more specific CS-no-US association in the case of extinction.

Given that the conditions that produce latent inhibition and extinction of conditioned responding are identical (CS-alone exposures) and given the similarity in the content of what is learned (CS-no-event vs. CS-no-US learning), it is of interest to consider how the two effects combine. Specifically, how does preexposure affect postextinction recovery of responding to a CS? Leung and Westbrook (2010, Experiment 6B) began to address this question using the compound test procedure (Rescorla, 2002). They showed that a CS subjected to both preexposure and extinction undergoes more recovery than does a CS just preexposed or just extinguished. In their procedure, one $\mathrm{CS}, \mathrm{A}$, was preexposed, and then both that $\mathrm{CS}$ and a second $\mathrm{CS}, \mathrm{C}$, were conditioned and extinguished (A, A+, A ; C+, C ). One week later, a third CS, B, was preexposed, and then both that CS and a fourth CS, D, were conditioned and extinguished (B, B+, B ; D+, D ). Shortly after the latter extinction, subjects were tested with compounds composed of $\mathrm{AD}$ and $\mathrm{BC}$. Note that both compounds consisted of a preexposed and extinguished CS (A and B), as well as a $\mathrm{CS}$ just extinguished (C and D). Moreover, both compounds consisted of a $\mathrm{CS}$ that had been trained remotely and was, therefore, subject to recovery (A and C), as well as a CS that had been trained recently (B and D). Critically, the compounds differed only in that one contained a CS undergoing recovery from both preexposure and extinction (A), while the other contained a CS undergoing recovery from just extinction $(\mathrm{C})$. The test revealed that subjects responded more to $\mathrm{AD}$ than to $\mathrm{BC}$. Thus, it was inferred that the $\mathrm{CS}, \mathrm{A}$, subjected to both preexposure and extinction had undergone greater recovery than the $\mathrm{CS}, \mathrm{C}$, just subjected to extinction.

The compound test procedure used by Leung and Westbrook (2010) permits the inference that the size of the recovery to a preexposed and extinguished CS is greater than that to a CS just extinguished. However, two issues remain to be clarified. The first issue pertains to the terminal levels of recovery to each CS. Greater recovery to a preexposed and extinguished CS may simply reflect that this CS enters the retention interval at a lower level than a CS just extinguished and that both CSs recover to the same terminal level of responding; however, it could also be that responding to a preexposed and extinguished CS recovers to a higher terminal level of responding than does a CS just extinguished. The second issue pertains to the conditions of recovery. The Leung and Westbrook procedure necessarily involved giving rats extra shocks prior to the compound test (i.e., during "recent" conditioning of B and D). Thus, the recovery they observed potentially reflects the combined effects of these additional shock experiences and the retention interval.

In light of these considerations, the present experiments had two aims. The first was to assess the terminal levels of recovery to a CS subjected to both preexposure and extinction versus a CS just subjected to extinction. The second aim was to investigate the importance of additional shock exposures in mediating differential recovery to the two CSs. Thus, in each experiment, rats were preexposed to a CS (A) and then to pairings of that CS, as well as a novel CS (B), with a shock US. Responding to each CS was then extinguished, and finally, rats were subjected to a test of both CSs after a delay. Exposure to unsignaled shock presentations in the delay interval was manipulated between experiments. The designs of the experiments are shown in Table 1.

\section{Experiment 1}

Experiment 1 investigated the effect of preexposure on conditioning, extinction, and recovery of fear responses. Rats were preexposed to one stimulus (designated A) and were conditioned to fear this stimulus, as well as a novel stimulus (designated B), and then responses to both CSs were extinguished. Following extinction training, rats received a US-alone exposure and were tested for reinstatement of conditioned responding to both CSs. Following a 5-day retention interval, rats were tested for recovery of conditioned responding to both CSs. The experiment was conducted and then replicated ( $n=16$ rats in each). There were no differences between the results of the two replications. Therefore, for convenience of exposition, the two replications are reported as a single experiment $(n=32)$. 
Table 1 Summary of designs in Experiments 1-4

\begin{tabular}{|c|c|c|c|c|c|c|}
\hline \multicolumn{7}{|c|}{ Experiment 1} \\
\hline Pre-exp & Cond & Ext & Reinst & Reinst Test & Retention & Recovery \\
\hline \multirow[t]{3}{*}{ A- } & $\mathrm{A}+$ & A- & & A- & & A- \\
\hline & & & + & & & \\
\hline & $\mathrm{B}+$ & B- & & B- & & B- \\
\hline \multicolumn{7}{|c|}{ Experiment 2} \\
\hline Pre-Exp & Cond & Ext & & Add Ext & Retention & Spont Rec \\
\hline \multirow[t]{2}{*}{ A- } & $\mathrm{A}+$ & A- & & A- & & A- \\
\hline & $\mathrm{B}+$ & B- & & B- & & B- \\
\hline \multicolumn{7}{|c|}{ Experiment 3} \\
\hline Pre-Exp & Cond & Ext & Reinst & & Retention & Recovery \\
\hline \multirow[t]{3}{*}{ A- } & $\mathrm{A}+$ & A- & & & & A- \\
\hline & & & + & & & \\
\hline & $\mathrm{B}+$ & B- & & & & B- \\
\hline \multicolumn{7}{|c|}{ Experiment 4} \\
\hline Pre-Exp & Cond & Ext & Retention & & Reinst & Recovery \\
\hline \multirow[t]{3}{*}{ A- } & $\mathrm{A}^{+}$ & A- & & & & A- \\
\hline & & & & & + & \\
\hline & $\mathrm{B}+$ & B- & & & & B- \\
\hline
\end{tabular}

Method

\section{Subjects}

Subjects were 32 experimentally naïve adult male Wistar rats (Rattus norvegicus) weighing between 400 and $470 \mathrm{~g}$ at the beginning of the experiment. They were obtained from a commercial supplier (Animal Research Centre, Perth, Australia) and were housed in groups of 8 in opaque plastic boxes $(67 \mathrm{~cm}$ length $\times 40 \mathrm{~cm}$ width $\times 22 \mathrm{~cm}$ height). The boxes were kept in an air-conditioned colony room maintained on a 12:12-h light:dark cycle (lights on at 7:00 a. $\mathrm{m}$.). Food and water were continuously available in the home cage during all phases of the experiment. Each rat was handled for 2-3 min each day for 4 days prior to the start of the experiment. All experimental procedures occurred between 9 a.m. and 6 p.m. The procedures were consistent with the ethical guidelines established by the American Psychological Association and were approved by the Animal Care and Ethics Committee of the University of New South Wales.

\section{Apparatus}

All phases of the experiment occurred in four identical chambers $(23.5 \mathrm{~cm}$ depth $\times 20.5 \mathrm{~cm}$ width $\times 19.5 \mathrm{~cm}$ height). The front and rear walls of these chambers, as well as the hinged lid, were constructed of clear Perspex, and the sidewalls were constructed of stainless steel. The floor of each chamber was composed of 2-mm-wide stainless steel rods spaced $13 \mathrm{~mm}$ apart (center to center). The floor of each chamber was $3 \mathrm{~cm}$ above a tray of paper pellet bedding material (Fibrecycle, Mudgeeraba, Australia) that was changed between rats. After each rat had been removed from the chambers, the floors, walls, and lids were cleaned with a $25 \%$ white vinegar (Coles Supermarkets, Australia) solution to eliminate any residue and provide a distinctive odor to the chambers.

Each chamber was enclosed in a sound- and lightattenuating shell. The inside walls of the shells were painted black. A white fluorescent tube and a speaker mounted at the back of each shell were used, respectively, for the presentation of a 30-s light CS (approximately 57 lux measured at the center of the chamber), flashing at a rate of 2.5/s during the otherwise dark session, and a $30-\mathrm{s}$ $1-\mathrm{kHz}$ spike wave tone $\mathrm{CS}$ with a rise/fall time of $10 \mathrm{~ms}$, measuring $75 \mathrm{~dB}$ (a scale) against a background noise of approximately $45 \mathrm{~dB}$, measured by a digital sound level meter (Dick Smith Electronics, Australia). In this and the subsequent experiments, the physical identity of all the CSs was fully counterbalanced, with half of the subjects preexposed to the tone and half to the flashing light. A constant-current shock generator, capable of delivering unscrambled AC $50 \mathrm{~Hz}$ to the floor of each chamber, was used for the presentation of a $0.5-\mathrm{mA}, 0.5$-s shock US. Illumination of each chamber was provided by an infrared light source $(940 \pm 25 \mathrm{~nm})$. A camera mounted on the back wall of each shell was used to record the behavior of each rat. Each camera was connected to a monitor and a DVD recorder located in another room of the laboratory. This room contained the computer that controlled stimulus presentations via appropriate software (LabView, National Instruments, Austin, TX). 


\section{Procedure}

The experiment involved seven phases: exposure to context, preexposure to the CS designated A, conditioning, extinction, US reexposure, reinstatement testing, and recovery testing.

Exposure to context On day 1, rats received a 30-min exposure to the experimental chambers in order to familiarize them with the context, thereby potentially reducing context conditioning.

Pre-exposure to $A$ On days 2-7, rats received daily sessions consisting of 20 nonreinforced exposures to the tone or flashing light (designated A). The first stimulus presentation in each session occurred $3.5 \mathrm{~min}$ after each rat had been placed in the chamber. Each stimulus presentation lasted $30 \mathrm{~s}$, and the average interval between stimulus presentations (defined as stimulus offset to stimulus onset) was $1.5 \mathrm{~min}$ (range: 30-150 s). After the final stimulus presentation in each session, rats remained in the chamber for a further 2 min before they were returned to their home cages. Thus, each preexposure session lasted $44 \mathrm{~min}$, and there were 120 exposures to the stimulus designated A across the 6 days of this phase.

Conditioning On days 8 and 9 rats received daily sessions consisting of four conditioning trials to each of the two CSs (i.e., eight trials in total to each $\mathrm{CS}$ over the 2 days of conditioning). The first CS was presented 2 min after each rat had been placed in the chamber. For half the rats, the session started with a familiar CS (designated A), while the remaining rats started the session with a novel CS (designated B). Thereafter, each CS was presented in an intermixed, pseudorandom order, with the constraint that the same CS could not occur on more than two consecutive trials. Each presentation of a CS lasted $30 \mathrm{~s}$ and coterminated with a footshock US (0.5-mA intensity and 0.5 -s duration). The average interval between trials was $4.5 \mathrm{~min}$ (range: $2.5-6.5 \mathrm{~min}$ ). After the final CS presentation in each session, rats remained in the chamber for a further $2 \mathrm{~min}$ before they were returned to their home cages. The average trial position for each CS was counterbalanced across the two conditioning sessions.

Extinction On days 10-13, rats received daily sessions consisting of 10 nonreinforced presentations of each CS (a total of 20 trials per session). The first CS presentation in each session occurred $3.5 \mathrm{~min}$ after the rats had been placed in the chambers. Each CS presentation lasted for $30 \mathrm{~s}$, and the average interval between $\mathrm{CS}$ presentations was $1.5 \mathrm{~min}$ (range: $30-150 \mathrm{~s}$ ). The order in which the two CSs were presented during extinction was subject to the same constraints as those described for conditioning.
Reinstatement On day 14, rats were placed in the chambers, and after $5 \mathrm{~min}$, a footshock was delivered that was identical to that used during conditioning. Rats remained in the chambers for an additional $5 \mathrm{~min}$ before being returned to their home cages (the session lasted for a total of $10.5 \mathrm{~min}$ ). On day 15 , rats were tested for freezing to A and $\mathrm{B}$ under conditions of extinction. The two CSs were presented in the same order and with the same constraints as during extinction.

Recovery Following the reinstatement test, rats remained in the colony room for the next 4 days, where they were handled for 2-3 min every second day. On day 20, rats were tested for recovery to both CSs. The details for this test session were identical to those described above for the reinstatement test.

\section{Scoring and statistical analyses}

The behavior of each rat over conditioning, extinction, reinstatement, and recovery test was videotaped and then scored to determine the levels of freezing behavior, which was defined as absence of all movements except those related to breathing (Fanselow, 1980). The levels of freezing were measured using a time-sampling procedure in which each rat's behavior was scored as freezing or not every $2 \mathrm{~s}$. Scoring began $30 \mathrm{~s}$ prior to stimulus onset (pre-CS interval), to provide indication of the fear to context, and terminated $30 \mathrm{~s}$ post-stimulus-onset. The number of occasions on which the behavior was scored as freezing was expressed as a percentage of the total number of observations (i.e., 15 samples for a 30-s CS). All the data were scored by the experimenter, who was blind to the rat's condition, and $25 \%$ of the test data were scored by an observer who was naïve as to the allocation of rats to counterbalanced conditions. The correlation between their scores was high $(\mathrm{r}>.90)$. Any discrepancies in scoring were resolved in favor of the naïve observer.

Freezing levels to the two CSs (A and B) in all phases of this and the subsequent experiments were analyzed using planned orthogonal contrasts which controlled the percontrast error rate at $\alpha=.05$ (Hays, 1973). With degrees of freedom $v 1=1$ and $v 2=31$, the $F_{\text {critical }}(1,31)$ value was 4.16 throughout all the phases of Experiment 1. A multivariate approach to repeated measures data was used (MANOVA; O'Brien \& Kaiser, 1985). For clarity, conditioning data were analyzed in single trials, whereas the data for extinction, reinstatement, and recovery were presented and analyzed in blocks of two trials. Paired $t$ tests were used to verify recovery of freezing between extinction and recovery test sessions (averaged over CS type), and where relevant, 
they were also used to investigate the source of significant interactions (trend $\times$ CS type).

Results

Conditioning Exposure to context, preexposure, and conditioning proceeded uneventfully. On the last conditioning session, the levels of freezing to A and B and the context were $53.33 \%, 57.71 \%$, and $60.52 \%$, respectively. Statistical analysis revealed that there was no difference in freezing to $\mathrm{A}$ and $\mathrm{B}$ across conditioning trials, $F(1,31)=1.84, p=.19$.

Extinction The mean percentage of freezing during each CS presentation, as well as $30 \mathrm{~s}$ prior to CS onset, over extinction training are shown in Fig. 1a. Inspection of this panel suggests that freezing to both CSs and the context decreased across extinction sessions. Inspection also suggests that, across extinction training, there was greater freezing during the presentation of the CSs (A and B) than during the context (pre-CS period). This was confirmed by statistical analysis, $F(1,31)=309.63, p<.05$. Statistical analysis also revealed a significant main effect of CS type, $F(1,31)=9.54$, $p<.05$, indicating that, across extinction, there was less freezing to A than to B. Analysis also revealed a significant linear decline in freezing across extinction blocks, $F(1,31)=185.03, p<.05$ indicating that responding to both CSs decreased across presentations. The linear trend $\times$ CS type interaction was not significant, $F(1,31)<1$.

Reinstatement The reinstatement data for both CSs and the pre-CS period are shown in Fig. 1b. Inspection of the panel also suggests that the unsignaled US presentation produced a reinstatement of conditioned responding, as compared with the level of freezing observed at the end of extinction, $t(31)=$ $9.76, p<.05$. Inspection of the data suggests that, across the reinstatement test, there was greater freezing to the CSs than to the context (pre-CS period). This was confirmed by statistical analysis, $F(1,31)=117.02, p<.05$. There was a significant linear trend, $F(1,31)=24.99, p<.05$, indicating that freezing responses to both CSs decreased in a linear fashion across the reinstatement test session. However, the main effect of CS type, $F(1,31)<1, p>.35$, and the linear trend $\times$ CS type interaction, $F(1,31)=2.21, p>.35$, were not significant.

Recovery The mean percentage of freezing during the presentation of both CSs and the pre-CS period in recovery tests are shown in Fig. 1c. Inspection suggests that there was substantial recovery of freezing to both CSs, as compared with the end of the reinstatement test, $t(31)=3.05, p<.05$. Furthermore, over the course of the recovery test, there was
Fig. 1 Mean percentage of freezing to each conditioned stimulus (CS; A and B) and in the 30 -s pre-CS interval during a four extinction sessions, $\mathbf{b}$ reinstatement test, and $\mathbf{c}$ recovery test in Experiment 1. Extinction training occurred over 4 days, and a reinstatement test (i.e., an additional, fifth extinction session) occurred 2 days after the last extinction session. Recovery testing occurred 4 days following the additional extinction session

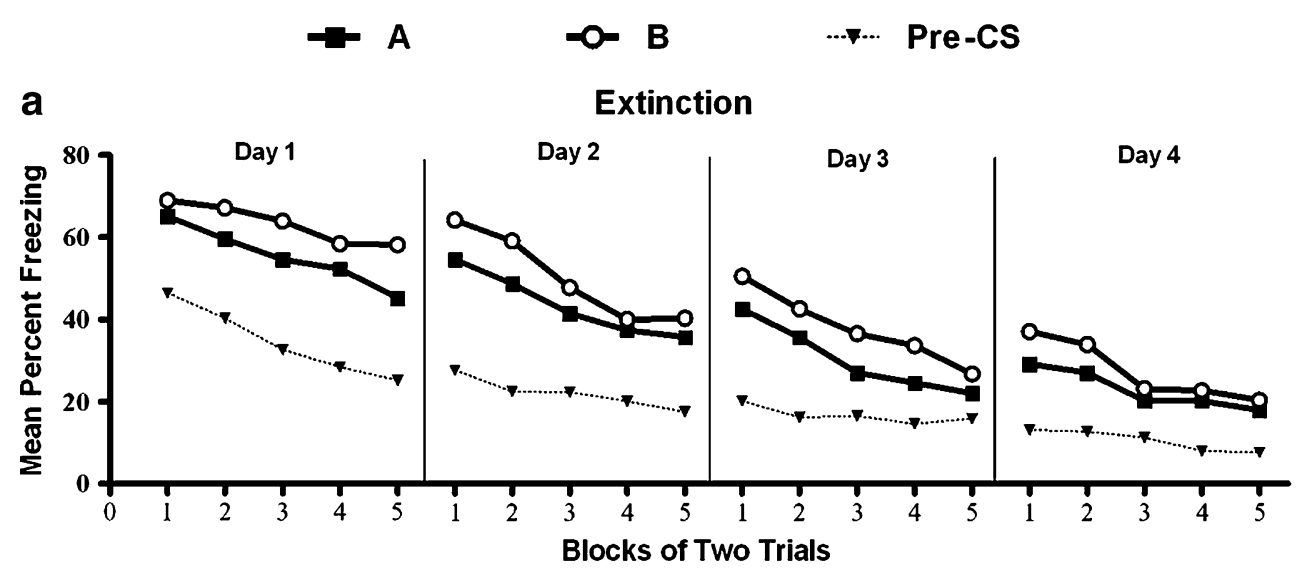

b

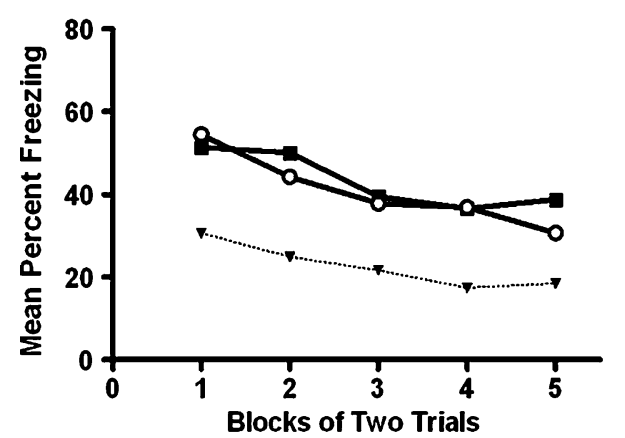

C Spontaneous Recovery

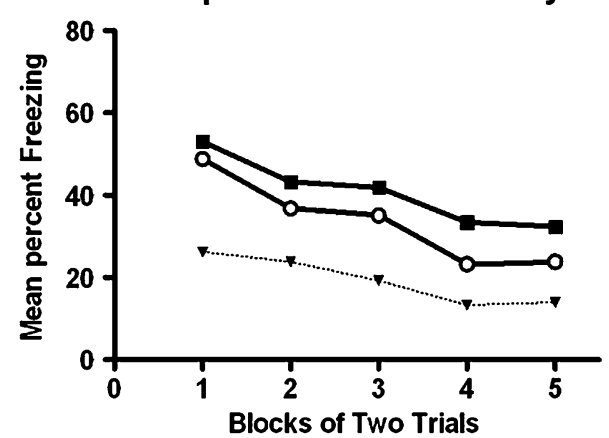


significantly greater freezing to the CSs than to the context (pre-CS period), $F(1,31)=123.508, p<.05$. Statistical analysis between the CSs revealed a significant main effect of CS type, $F(1,31)=13.33, p<.05$, confirming that the levels of freezing were greater for A than for B. Furthermore, the level of freezing showed a linear decrease over the course of the test, $F(1,31)=27.55, p<.05$. The CS type $\times$ linear trend interaction was not significant, $F(1,31)<1$, indicating that there were no differences in the rate of decrease of responding as a function of CS type.

\section{Discussion}

Experiment 1 failed to show an effect of preexposure on acquisition of conditioned freezing responses. This is likely due to high overall levels of freezing during fear conditioning that obscured differences in conditioning strength between the preexposed (A) and nonpreexposed (B) CSs. However, less freezing to A than B (i.e., latent inhibition) was observed during extinction once overall levels of freezing decreased. Preexposure did not affect the magnitude of reinstatement, with an unsignaled US presentation reinstating conditioned responding to both CSs to an equal level. During the delayed test, there was a reversal of the latent inhibition pattern observed during extinction, with greater recovery of conditioned responses to A than to B.

The differential recovery to the CSs in the delayed test confirms and extends Leung and Westbrook's (2010) findings in showing that responding to a preexposed and extinguished CS recovers to a higher terminal level than does a CS just extinguished. However, as in the Leung and Westbrook study, it is not clear whether the differential recovery can be attributed to the passage of time alone, or whether the greater recovery to $A$ than to $B$ was precipitated by experience of unsignaled shock between the start of extinction and test. Accordingly, the following experiments were designed to identify the importance of unsignaled shock presentation in producing greater recovery to the preexposed than to the nonpreexposed CS.

\section{Experiment 2}

The design of Experiment 2 is illustrated in Table 1. Experiment 2 differed from Experiment 1 only in that presentation of the reinstating shock after extinction training was omitted. If the result observed in Experiment 1 was the consequence of the passage of time alone, we would again expect to observe greater recovery to the preexposed and extinguished CS, A, than to the CS just extinguished, B. However, if additional shock exposures were critical to the result observed in Experiment 1, we would expect equal recovery to $\mathrm{A}$ and $\mathrm{B}$.

Method

\section{Subjects and apparatus}

The subjects were 16 experimentally naïve adult male rats of the same weight and from the same source as those in Experiment 1. Rats were housed and maintained in the manner described for Experiment 1. The apparatus was the same as that used in Experiment 1. The CSs were again counterbalanced as described previously.

\section{Procedure}

The procedure used in this experiment was identical to that used in Experiment 1, except for the absence of a reinstating shock in the interval between initial extinction after conditioning and additional extinction (included to match the reinstatement test in Experiment 1). All rats were exposed to the context (day 1), preexposed to A (days 2-7), conditioned (days 8 and 9), and extinguished (days 10-13) in the manner described in Experiment 1.

Additional (fifth) extinction (matching the reinstatement test from Experiment 1) On day 15, rats received an additional extinction session to each of the two CSs. This additional session was identical to the reinstatement test administered in Experiment 1.

Recovery On day 20, rats were tested for recovery to both $\mathrm{CSs}$ in the manner described in Experiment 1.

\section{Scoring and statistical analyses}

Scoring of freezing behavior and statistical analyses were the same as in the previous experiment. With degrees of freedom $v 1=1$ and $v 2=15$, the $F_{\text {critical }}(1,15)$ value was 4.54 throughout all the phases of the experiment.

\section{Results}

Conditioning Exposure to the context, preexposure, and conditioning proceeded uneventfully. On the last conditioning session, the levels of freezing to A, B, and the context were $45.83 \%, 50.83 \%$, and $52.50 \%$, respectively. Statistical analysis revealed that there was no difference in freezing to $\mathrm{A}$ and $\mathrm{B}$ across conditioning trials, $F(1,15)<1$.

Extinction The mean percentage of freezing during CS presentations, as well as in the pre-CS periods, over extinction 
training in Experiment 2 are shown in Fig. 2a. Inspection of this panel suggests that responding to both CSs and the context decreased across extinction training and that there was much greater freezing to the CSs than to the context, which was confirmed by statistical analysis, $F(1,15)=$ $228.30, p<.05$. Furthermore, analysis revealed that there was less freezing to $\mathrm{A}$ than to $\mathrm{B}, F(1,15)=5.00, p<.05$, and that the freezing to both CSs decreased across extinction training, $F(1,15)=95.06, p<.05$. The trend $\times$ CS type interaction was not significant, $F(1,15)<1$, indicating that there was no difference in the rate of decline of freezing responses to $\mathrm{A}$ and $\mathrm{B}$.

Additional (fifth) extinction The mean percentage of freezing to $\mathrm{A}$ and $\mathrm{B}$ and the context during the additional extinction session in Experiment 2 are shown in Fig. 2 b. Inspection of this panel suggests that at the onset of the additional extinction session, there was a recovery of freezing responses to both CSs, as compared with the levels of freezing at the end of the initial extinction, $t(15)=$ $3.46, p<.05$. Inspection also suggests that there were greater levels of freezing during the presentation of the two CSs than during the pre-CS period, $F(1,15)=72.95, p<$ .05. Furthermoe, statistical analysis between A and B confirmed that freezing to both CSs declined across the additional extinction session, $F(1,15)=5.27, p<.05$; however, the main effect, $F(1,15)<1$, and the interaction contrast, $F(1,15)=1.89, p>.15$, were not significant.

Recovery The mean percentage of freezing across CS presentations, as well as in the pre-CS periods, during the recovery test in Experiment 2 are shown in Fig. 2c. Inspection suggests that the levels of freezing to both $\mathrm{A}$ and $\mathrm{B}$ recovered following the passage of time, which was confirmed by statistical analysis, $t(15)=2.56, p<.05$. Also, from inspection, it is evident that the pre-CS levels of freezing throughout the session were lower than the CS levels, which was confirmed by statistical analysis, $F(1,15)=33.49, p<$ .05 . However, the data of most interest are the responding to $\mathrm{A}$ and $\mathrm{B}$ across the session. Statistical analysis confirmed a significant linear trend, $F(1,15)=17.53, p<.05$, indicating that freezing decreased linearly across the nonreinforced presentations of the CS. However, the main effect, $F(1,15)=$ $2.76, p>.10$, and the interaction contrast, $F(1,15)<1$, were not significant.

\section{Discussion}

Experiment 2 confirmed that a preexposed CS evokes less conditioned responding than does a nonpreexposed CS across extinction training. However, following extinction
Fig. 2 Mean percentage of freezing to each conditioned stimulus (CS; A and B) and in the 30 -s pre-CS interval during a four extinction sessions, b additional extinction (equivalent to a reinstatement test from Experiment 1), and c spontaneous recovery test in Experiment 2. Extinction training occurred over 4 days, and an additional (fifth) extinction session occurred 2 days after the last extinction session. Spontaneous recovery testing occurred 4 days following the additional extinction session

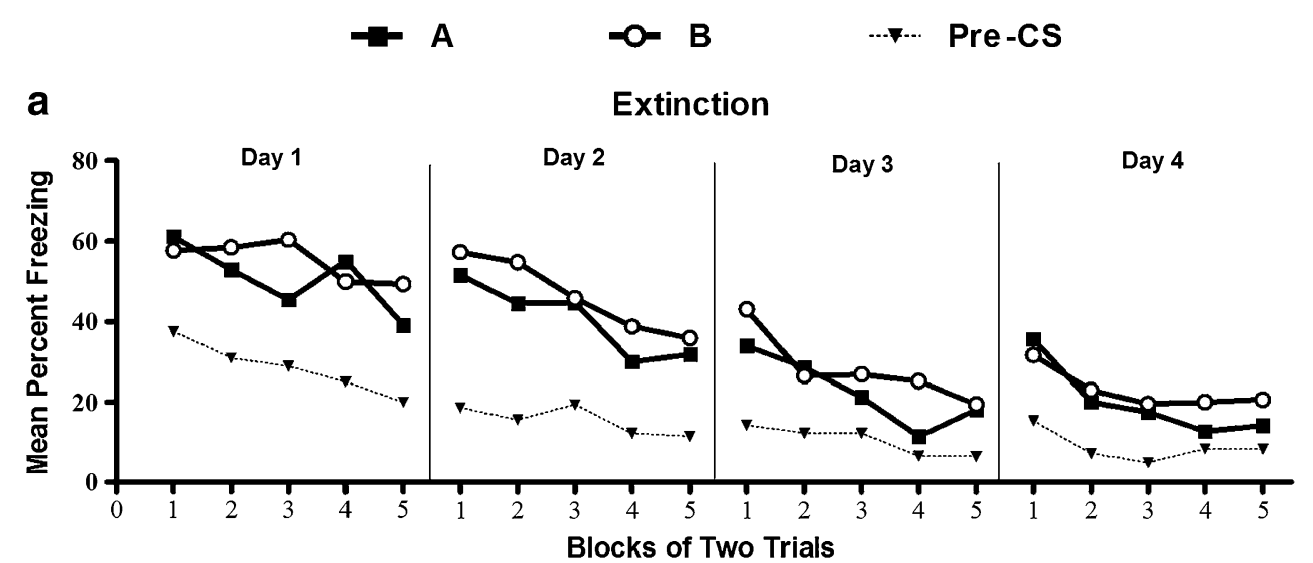

b

C Spontaneous Recovery

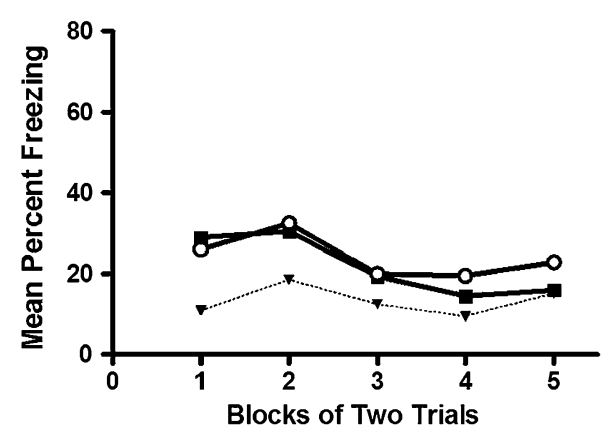

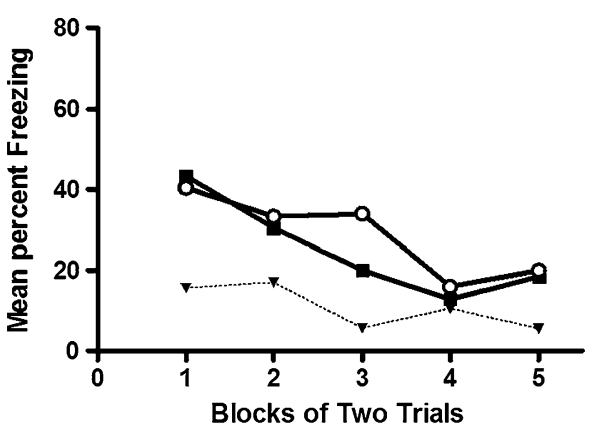


training, there was no evidence of differential recovery of responding to the two CSs. Although based on a null result, this suggests that additional experience of shock between extinction and test is critical in establishing greater recovery to the preexposed and extinguished CS than to the CS just extinguished. The following experiments examined whether the locus of the additional shock presentation in the extinction-test interval is important in producing greater recovery to $\mathrm{A}$ than to $\mathrm{B}$.

\section{Experiment 3}

Experiment 3 examined the effect of a postextinction US reexposure on recovery of conditioned fear responses to A and $\mathrm{B}$. The present experiment differed from Experiment 1 only in that rats received no additional nonreinforced exposures to $\mathrm{A}$ and $\mathrm{B}$ subsequent to the US-alone exposure but, rather, were tested for recovery 6 days later. We again anticipated greater recovery of responding to A than to B.

\section{Method}

\section{Subjects and apparatus}

The subjects were 16 experimentally naïve adult male rats of the same weight and from the same source as in the previous experiments. Rats were housed and maintained in the manner described for Experiment 1. The apparatus was the same as that used in Experiment 1. The CSs were counterbalanced in the manner described previously.

\section{Procedure}

The procedure in Experiment 3 differed from that in Experiment 1 only in the absence of an additional extinction session (the reinstatement test in Experiment 1) following US reexposure. All subjects were exposed to the context (day 1), preexposed to A (days 2-7), conditioned (days 8 and 9), and extinguished (days 10-13) in the manner described in Experiment 1.

Shock exposure On day 14, rats were placed in the chambers. After $5 \mathrm{~min}$ had elapsed, they were given a single US exposure of the same intensity and duration as that used in Experiment

1. After a further $5 \mathrm{~min}$, rats were returned to their home cages. Rats remained in their home cages for the next 6 days.

Recovery On day 20, subjects were tested for recovery to both CSs in the manner described in Experiment 1.

\section{Scoring and statistical analyses}

Scoring of freezing behavior and statistical analyses were the same as those previously described. As in Experiment 2 , the $F_{\text {critical }}(1,15)$ value was 4.54 throughout all the phases of the experiment.

Results

Conditioning Exposure to the context, preexposure, and conditioning proceeded uneventfully. On the last conditioning session, the levels of freezing to A, B, and the context were $54.17 \%, 43.33 \%$, and $42.30 \%$, respectively. Statistical analysis between the CSs revealed that there was no difference in freezing to $\mathrm{A}$ and $\mathrm{B}$ across conditioning trials, $F(1,15)<1$.

Extinction The mean percentage of freezing during CS presentations and pre-CS periods over extinction training in Experiment 3 are shown in Fig. 3a. Inspection of the data suggests that there was much greater freezing during presentations of the CSs than during pre-CS

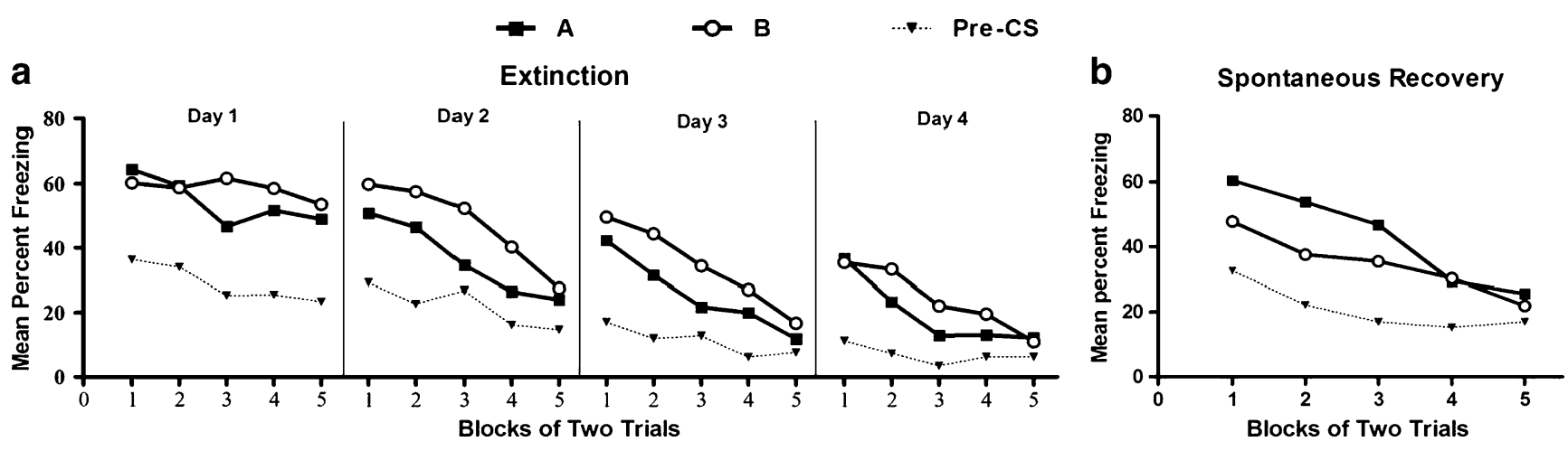

Fig. 3 Mean percentage of freezing to each conditioned stimulus (CS; $\mathrm{A}$ and $\mathrm{B}$ ) and in the 30-s pre-CS interval during a four extinction sessions and $\mathbf{b}$ recovery test in Experiment 3. Extinction training occurred over 4 days, and recovery testing occurred 6 days following the last extinction session and 5 days following US reexposure 
periods, $F(1,15)=125.49, p<.05$. Of particular interest are freezing responses to the two CSs across extinction training. Statistical analysis revealed a significant main effect of CS type, $F(1,15)=5.44, p<.05$, as well as a significant linear trend, $F(1,15)=71.36, p<.05$, indicating that across extinction training, there was less freezing to A than to B and that responding to both CSs decreased throughout extinction training. However, the trend $\times$ CS type interaction was not significant, $F(1,15)<1$.

Recovery The mean percentage of freezing during CS presentations and pre-CS periods are shown in Fig. $3 \mathrm{~b}$. Inspection of the data suggests a marked recovery of freezing responses to both CSs. This was confirmed by a statistical analysis that revealed that there was recovery of freezing responses to both CSs, as compared with the end of extinction training, $t(15)=10.85, p<.05$. Furthermore, analysis between the levels of freezing to the CSs and the context revealed higher levels of freezing during the CSs than during the context, $F(1,15)=129.87, p<.05$. The most important analysis compared the differences in levels of freezing to $\mathrm{A}$ and $\mathrm{B}$. This analysis confirmed that there was greater freezing to $\mathrm{A}$ than to $\mathrm{B}, F(1,15)=6.72, p<$ .05 . The analysis also revealed a significant linear trend, $F$ $(1,15)=36.31, p<.05$, indicating that freezing responses to both CSs decreased across the session, but the trend $\times$ CS type interaction contrast failed to reach a conventional level of significance, $F(1,15)=4.07, p=.06$.

\section{Discussion}

As in the previous experiments, subjects in Experiment 3 displayed lower levels of freezing to A than to B across extinction training, consistent with the occurrence of latent inhibition. More important, Experiment 3 demonstrated greater recovery of conditioned fear responses to a preexposed than to a nonpreexposed CS when subjects received a US-alone presentation before they entered the delay. This strongly supports the view that additional postextinction shock experience is a crucial factor in producing differential recovery to a preexposed and extinguished CS and a CS just extinguished. The effects of the additional shock experience and the passage of time on response recovery appear to combine, resulting in greater recovery to A than to B.

\section{Experiment 4}

Experiment 4 further examined the effect of a postextinction US reexposure on recovery of conditioned fear responses to $\mathrm{A}$ and $\mathrm{B}$. The present experiment differed from Experiment 3 only in the timing of US reexposure, relative to extinction and testing. That is, rats were reexposed to the US 6 days after the final extinction session and were tested for recovery the following day. On the basis of the previous results, we again anticipated that US reexposure would lead to greater recovery of fear responses to $\mathrm{A}$ than to $\mathrm{B}$ during the recovery test.

\section{Method}

\section{Subjects and apparatus}

The subjects were 16 experimentally naïve adult male rats of the same weight and from the same source as in the previous experiments. Rats were housed and maintained in the manner described for Experiment 1. The apparatus was the same as that used in Experiment 1. The CSs were counterbalanced in the manner described previously.

\section{Procedure}

The procedure in Experiment 4 differed from that in Experiment 3 only in that rats were reexposed to the US 6 days after the final extinction session and were tested for recovery 1 day later. All the subjects were exposed to the context (day 1), preexposed to A (days 2-7), conditioned (days 8 and 9), and extinguished (days 10-13) in the manner described in Experiment 1.

Delay and shock exposure On days 14-18 rats remained in their home cages. On day 19, rats were placed in the chambers. After $5 \mathrm{~min}$ had elapsed, they were given a single US exposure of the same intensity and duration as that used in Experiment 1. After a further $5 \mathrm{~min}$, rats were returned to their home cages.

Recovery test On day 20, rats were tested for recovery to both CSs in the manner described in Experiment 1.

\section{Scoring and statistical analyses}

Scoring of freezing behavior and statistical analyses were the same as previously described. As in Experiment 2, the $F_{\text {critical }}(1,15)$ value was 4.54 throughout all the phases of the experiment.

Results

Conditioning Exposure to the context, preexposure, and conditioning proceeded uneventfully. On the last conditioning session, the levels of freezing to A, B, and the context were $32.4 \%, 36.4 \%$, and $45.4 \%$, respectively. Statistical analysis 
between the CSs revealed that there was no difference in freezing to $\mathrm{A}$ and $\mathrm{B}, F(1,15)<1$.

Extinction The mean percentage of freezing during CS presentations and pre-CS periods over extinction training in Experiment 4 are shown in Fig. 4a. Inspection of the data suggests that there was much greater freezing during presentations of the CSs than during pre-CS periods, $F(1$, $15)=35.77, p<.05$. Of particular interest are freezing responses to the two CSs across extinction training. Statistical analysis revealed that the main effect of CS type was not significant, $F(1,15)=3.79, p=.07$. However, there was a significant linear trend across sessions, $F(1,15)=$ $102.78, p<.05$, and a significant trend $\times$ CS type interaction, $F(1,15)=6.37, p<.05$. These results indicate that freezing to both CSs decreased across extinction training but that the rate of decrease differed for the two CSs, such that there was less freezing to $\mathrm{A}$ than to $\mathrm{B}$ in sessions 1 and 3 (smaller $t(15)=2.26, \mathrm{p}<.05)$, but not in sessions 2 and 4 (larger $t(15)=1.10, \mathrm{p}>.2)$.

Recovery test The mean percentage of freezing during CS presentations and pre-CS periods are shown in Fig. 4b. Inspection of the data suggests a recovery of freezing responses to both CSs and the context. This was confirmed by a statistical analysis that revealed greater overall freezing in the test session than in the final extinction session, $t(15)=6.30, p<.05$. Furthermore, analysis between the levels of freezing to the CSs and the context revealed higher levels of freezing during the CSs than during the context, $F(1,15)=10.04, p<.05$. The most important analysis compared the differences in levels of freezing to $\mathrm{A}$ and $\mathrm{B}$. This analysis confirmed that there was greater freezing to $\mathrm{A}$ than to $\mathrm{B}, F(1,15)=21.434, p<$ .05 . The analysis also revealed a significant linear trend, $F$ $(1,15)=17.6, p<.05$, indicating that freezing responses to both CSs decreased across the session. The trend $\times \mathrm{CS}$ type interaction contrast did not reach the conventional level of significance, $F(1,15)=4.09, p=.06$.

\section{Discussion}

Experiment 4 again showed less freezing to A than to B during extinction. It replicated the findings in Experiments 1 and 3 of greater recovery of conditioned fear responses to a preexposed and extinguished $\mathrm{CS}$ than to a CS just extinguished when the postextinction US interval incorporated a single US presentation. However, it has critically shown that greater postextinction recovery to $\mathrm{A}$ than to $\mathrm{B}$ does not depend on the timing of US reexposure, relative to extinction training and testing. That is, US reexposure in the delay interval leads to greater recovery to A than to $\mathrm{B}$ regardless of whether this exposure occurs early (1 day in Experiment 3) or late (6 days in Experiment 4) in the interval between extinction and test.

\section{General discussion}

The present experiments showed that conditioned freezing to a preexposed CS, A, exhibited more loss across extinction than did that to a nonpreexposed CS, B, thus demonstrating latent inhibition. Each experiment went on to investigate postextinction recovery of responding to $\mathrm{A}$ and $\mathrm{B}$. When rats were reexposed to the US $24 \mathrm{~h}$ after the final extinction session and tested the following day, there was no evidence for differential reinstatement of responding to A versus B (Experiment 1). Similarly, when rats were tested 1 week after the final extinction session, there was no differential spontaneous recovery to A versus B (Experiment 2). However, differential recovery to A versus B was observed when the "reinstatement" and delay manipulations were combined. That is, delayed tests revealed greater recovery to A than to B when the extinction-test interval included US reexposure. This advantage for the preexposed CS

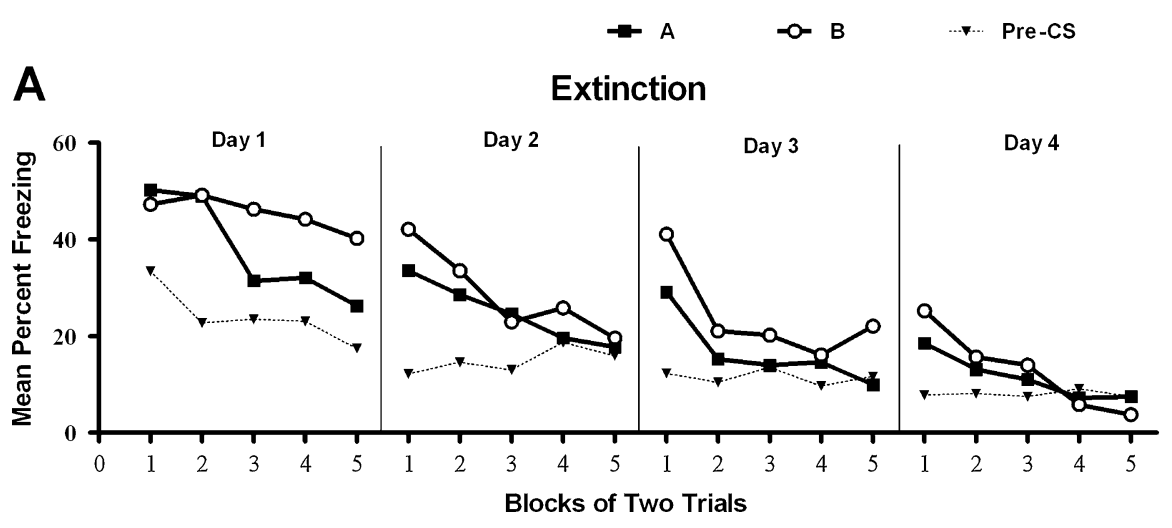

B

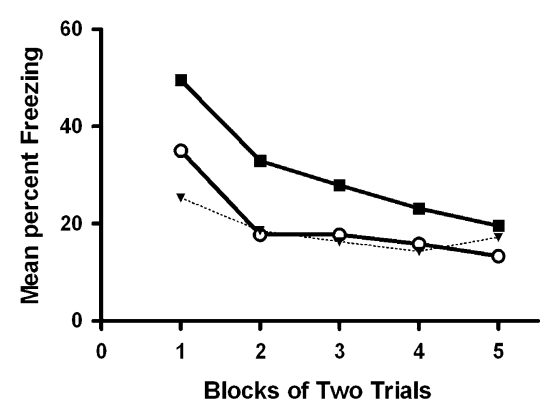

Fig. 4 Mean percentage of freezing to each conditioned stimulus (CS; $\mathrm{A}$ and $\mathrm{B}$ ) and in the 30 -s pre-CS interval during a four extinction sessions and $\mathbf{b}$ recovery test in Experiment 4. Extinction training occurred over 4 days, and recovery testing occurred 6 days following the last extinction session and 1 day following US reexposure 
was evident when US reexposure occurred either early (Experiments 1 and 3) or late (Experiment 4) in the delay interval. Thus, these experiments show that the effects of a postextinction US-alone exposure and a delay interval combine to produce greater recovery to $\mathrm{A}$ than to $\mathrm{B}$ at test.

As was noted in the introduction, Leung and Westbrook (2010) used the compound test procedure to show greater recovery of responding to a preexposed and extinguished CS than to a CS just preexposed or just extinguished. The present results extend this finding in showing that it is not just that a preexposed and extinguished CS enters the delay interval at a lower level and that both CSs recover to the same terminal level of responding. Instead, these results unambiguously show that a preexposed and extinguished CS recovers to a higher terminal of responding than does a CS just extinguished. In the introduction, we also noted that Leung and Westbrook's use of the compound test procedure necessitated additional shock exposures during conditioning of the stimuli that were not subject to recovery. The present results strongly imply that these additional shock exposures were critical in establishing greater recovery of responding to the preexposed and extinguished CS, thereby challenging Leung and Westbrook's interpretation of their findings in terms of spontaneous recovery alone. However, this prompts the following question: Why are both US reexposure and delay manipulations needed in order to uncover the advantage for $\mathrm{A}$ over $\mathrm{B}$ in postextinction recovery?

At present, we can only speculate as to why this is the case. One possibility is that manipulations of the context come to have a more profound effect on CSs that were preexposed in that context. For instance, it is widely accepted that preexposure to the CS-to-be results in both context-CS and CS-context associations, while unsignaled shock exposures produce context-US associations. Consequently, the additional shock exposure may have resulted in mediated conditioning of the preexposed CS, and hence, we observed greater recovery to A than to B. However, the story cannot be as simple as this, since we observed equal responding to $\mathrm{A}$ and $\mathrm{B}$ during the immediate reinstatement test in Experiment 1. Something must have changed between the time of this test and the later recovery test. One possibility is that nonreinforced experiences of $\mathrm{B}$ during extinction training produced a transient context-B association that allowed some mediated conditioning of B to occur. Hence, when the test occurred immediately after US reexposure, there was equal recovery of responding to $\mathrm{A}$ and $\mathrm{B}$. But since there were many more nonreinforced exposures to $\mathrm{A}$ than to $\mathrm{B}$ in the context (160 exposures to $\mathrm{A}$ vs. 40 exposures to $B$ at the end of extinction training), the passage of time may have had more of a disruptive effect on the context-B association than on the robust context-A association. Hence, US reexposure alone produced equal reinstatement of responding to $\mathrm{A}$ and $\mathrm{B}$; the passage of time alone produced equal recovery of responding to $\mathrm{A}$ and $\mathrm{B}$; but including US reexposure in the delay interval allowed for better expression of mediated conditioning to A than to $\mathrm{B}$ at the end of the delay and, thus, more responding to $\mathrm{A}$ than to B. This account of the present findings has a number of implications. First, it predicts that greater recovery to A than to B will be observed only when US reexposure occurs in the context of preexposure. Second, it predicts that extinction training is not necessary for the effect to be observed beyond a lowering of response rates below ceiling. Third, it predicts that US reexposure at the start of the delay interval will be less effective in producing better recovery to $\mathrm{A}$ than to $\mathrm{B}$ than US reexposure at the end of the delay interval will be, due to differential mediated conditioning of $\mathrm{A}$ and $\mathrm{B}$ in the latter, but not the former, case. These predictions remain to be verified.

Differential expression of mediated conditioning to A and $\mathrm{B}$ may not be the only mechanism by which greater recovery to $\mathrm{A}$ than to $\mathrm{B}$ occurs. Another possibility is that the mechanisms described in the Pearce and Hall (1980) model - and more recently, in the modified version of this model by Hall and Rodriguez (2010) - also contribute to this result. This model holds that the associability of a CS is inversely related to how well it predicts the outcome, such that CSs followed by nonsurprising outcomes have lower associability than do CSs followed by surprising outcomes. By this reasoning, nonreinforced presentations of A during extinction are less surprising than nonreinforced presentations of B, because of A's history of such presentations during preexposure. This would mean that the associability of A declines more rapidly than that of $\mathrm{B}$ during extinction. The faster decline in associability to A than to B has several implications. First, responding will cease more rapidly to A than to $\mathrm{B}$, and second, there will be less learning of the new A-no-US association than of the B-no-US association. A final implication of the faster decline in associability to A than to $\mathrm{B}$ across extinction is that there will be less erasure of the A-US association than of the B-US association. Even if it were assumed that the A-US association was weaker than the B-US associations at the outset of extinction (Reiss \& Wagner, 1972; Rescorla, 1971, 2002), the Hall-Rodriguez model allows greater residual conditioning strength (less loss of strength) for A than for B at the end of extinction. Thus, although the Hall-Rodriguez model offers no explanation as to why both US reexposure and delay manipulations are necessary, it generally accommodates the present findings of greater recovery to A than to $\mathrm{B}$ in the delayed tests.

In summary, the present experiments have confirmed a previous report that responding to a preexposed and extinguished CS undergoes greater recovery than does responding to a CS that has been just extinguished (Leung \& Westbrook, 
2010). They have extended this previous finding in showing that responding to a preexposed and extinguished CS recovers to a higher terminal level than does responding to a CS just extinguished and that postextinction US reexposure is critical to this result. This result may reflect the fact that postextinction changes in context value more strongly affect performance to a preexposed and extinguished CS than to a CS just extinguished, since the former CS is more strongly associated with the context. The mechanisms described in the Hall and Rodriguez (2010) model may also contribute to the advantage for the preexposed and extinguished CS in postextinction recovery.

\section{References}

Bouton, M. E. (1991). Context and retrieval in extinction and in other examples of interference in simple associative learning. In L. Dachowski \& C. F. Flaherty (Eds.), Current topics in animal learning: Brain, emotion, and cognition (pp. 25-53). Hillsdale, NJ: Erlbaum.

Bouton, M. E. (1993). Context, time and memory retrieval in the interference paradigms of Pavlovian learning. Psychological Bulletin, 114, 80-99.

Fanselow, M. S. (1980). Conditioned and unconditioned components of post-shock freezing. Pavlovian Journal of Biological Sciences, $15,177-182$

Hall, G., \& Honey, R. C. (1989). Attenuation of latent inhibition after compound pre-exposure: Associative and perceptual explanations. The Quarterly Journal of Experimental Psychology, 41B, 355-368.

Hall, G., \& Rodriguez, G. (2010). Associative and nonassociative processes in latent inhibition: An elaboration of the Pearce-Hall model. In R. Lubow \& I. Weiner (Eds.), Latent inhibition: Cognition, neuroscience, and applications to schizophrenia. New York: Cambridge University Press.

Hays, W. L. (1973). Statistics for the social science (2nd ed.). New York: Holt, Rinehart \& Winston.

Holmes, N., \& Harris, J. A. (2010). Latent inhibition. In C. Mitchell \& M. Le Pelley (Eds.), Attention and associative learning: From brain to behavior (pp. 99-130). Oxford: Oxford University Press.
Killcross, A. S., \& Robbins, T. W. (1993). Differential effects of intra-accumbens and systemic amphetamine on latent inhibition using an on-baseline, within-subject conditioned suppression paradigm. Psychopharmacology, 110, 479-489.

Killcross, A. S., \& Balleine, B. (1996). Role of primary motivation in stimulus preexposure effects. Journal of Experimental Psychology: Animal Behavior Processes, 22, 32-42.

Leung, H. T., \& Westbrook, R. F. (2010). Increased spontaneous recovery with increases in conditioned stimulus alone exposures. Journal of Experimental Psychology: Animal Behavior Processes, 36, 354-367.

Lubow, R. E., \& Moore, A. U. (1959). Latent inhibition: Effects of frequency of nonreinforced preexposure to the conditioned stimulus. Journal of Comparative and Physiological Psychology, $52,415-419$.

Lubow, R. E., Weiner, I., \& Schnur, P. (1981). Conditioned attention theory. In G. H. Bower (Ed.), The psychology of learning and motivation, 15, 1-49. New York: Academic Press.

O'Brien, R. G., \& Kaiser, M. K. (1985). MANOVA method for analyzing repeated measures designs: An extensive primer. Psychological Bulletin, 97, 316-333.

Pearce, J. M., \& Hall, G. (1980). A model for Pavlovian learning: Variations in the effectiveness of conditioned but not unconditioned stimuli. Psychological Review, 87, 532-552.

Reiss, S., \& Wagner, A. R. (1972). CS habituation produces a 'latent inhibition effect' but no active 'conditioned inhibition. Learning and Motivation, 3, 237-245.

Rescorla, R. A. (1971). Summation and retardation tests of latent inhibition. Journal of Comparative and Physiological Psychology, $75,77-81$.

Rescorla, R. A. (2002). Savings test: Separating differences in the rate of learning from differences in initial levels. Journal of Experimental Psychology: Animal Behavior Processes, 28, 369-377.

Westbrook, R. F., \& Bouton, M. E. (2010). Latent inhibition and extinction: Their signature phenomena and the role of prediction error. In R. Lubow \& I. Weiner (Eds.), Latent inhibition: Cognition, neuroscience, and applications to schizophrenia (pp. 23-39). New York: Cambridge University Press.

This research was funded by Australian Research Council Discovery Grant DP1096570. 